\title{
Narrow-band full Stokes polarimetry of small structures on the Sun with speckle methods
}

\author{
N. Bello González and F. Kneer
}

\begin{abstract}
Institut für Astrophysik, Friedrich-Hund-Platz 1, 37077 Göttingen, Germany
\end{abstract}
e-mail: [nazaret;kneer]@astro.physik.uni-goettingen.de

Received 29 August 2007 / Accepted 19 December 2007

\section{ABSTRACT}

\begin{abstract}
Aims. For the detection and the study of small-scale magnetic fields on the Sun, it is important to obtain observations with both high spatial resolution and high polarimetric sensitivity.

Methods. A second narrow-band etalon and a full Stokes polarimeter, based on ferroelectric liquid crystals, were implemented in the two-dimensional "Göttingen" Fabry-Perot spectrometer/polarimeter at the Vacuum Tower Telescope, Observatorio del Teide/Tenerife. First observations with the Fe I $6173 \AA$ A line and their data analysis with speckle methods are described.

Results. The new polarimeter yields magnetograms of a field of view of $31^{\prime \prime} \times 52^{\prime \prime}$ with minimised seeing induced signals and without spurious signals as from the use of beam-splitting calcites. The achieved spatial and temporal resolution are 0.'30-0!35 and $36 \mathrm{~s}$, respectively. With a detection limit for the field strength of $16 \mathrm{G}(\widehat{=} 3 \sigma)$, a polarimetric sensitivity of $7-8 \times 10^{15} \mathrm{Mx}$ is obtained. Examples of intensity maps, Dopplergrams, and magnetograms from quiet and active regions on the Sun are discussed. Some of the results on solar magnetism are on 1) a pore with small-scale structure where we find a region with very low temperature gradient, 2) polar faculae with strong magnetic field signals and weaker signals in other areas surrounding them, and 3) small-scale inter-network magnetic fields with area fillings in the resolution elements of the order of 0.15 .
\end{abstract}

Key words. Sun: magnetic fields - techniques: high angular resolution - techniques: polarimetric

\section{Introduction}

Small-scale magnetic fields represent important ingredients for the structure and the dynamics of the atmosphere of the Sun (and of astrophysical plasmas in general). For works on the quiet Sun magnetism, we refer to Schrijver \& Title (2003), Trujillo Bueno et al. (2004), Khomenko et al. (2005), and Domínguez Cerdeña et al. (2006), and on the complexity of magnetic fields in sunspots to Title et al. (1993), Westendorp Plaza et al. (2001), Bello González et al. (2005), Borrero et al. (2006), Scharmer \& Spruit (2006), and Borrero (2007). See also the literature cited in these works. Thus, efforts are undertaken worldwide to perform spectro-polarimetry of the solar spectrum with high polarimetric sensitivity and with high spatial, spectral, and temporal resolution. We mention, among many others, the Tenerife Infrared Polarimeter TIP (Martínez Pillet et al. 1999), which has been upgraded to TIP 2 during the past years (Collados et al. 2007), the Zurich Imaging Polarimeter ZIMPOL (Gandorfer 2003), the successfully launched Solar-B mission, now called HINODE (Ichimoto et al. 2004) ${ }^{1}$, and the $1.5 \mathrm{~m}$ solar telescope GREGOR presently under construction (Volkmer et al. 2007).

The "Göttingen" two-dimensional (2D) spectrometer was built and mounted in the Vacuum Tower Telescope (VTT) at the Observatorio del Teide/Tenerife during the early 1990s (Bendlin et al. 1992; Bendlin \& Volkmer 1995). Its efficiency was substantially improved in 2005 (Puschmann et al. 2006, see also references to earlier work therein). The spectrometer is based on two Fabry-Perot interferometers (FPIs) which transmit a narrow-band 2D field of view (FOV). Wavelength scanning is

${ }^{1}$ See also the HINODE web pages at http://solarb.msfc .nasa. gov/ accomplished by computer controlled changes of the etalon spacings. The FPI spectrometer performed well with respect to its high throughput (Kneer 2007, as all FPI spectrometers) and with respect to its spatial and temporal resolution. Magnetograms were also obtained with a Stokes $V$ polarimeter (Volkmer et al. 1995; Koschinsky et al. 2001; Bello González et al. 2005).

In April 2007, a further narrow-band etalon was added to the spectrometer, running in tandem with the already existing narrow-band etalon. The spectral purity and resolution was substantially improved.

Most importantly, the earlier polarimetry of the spectrometer had its drawbacks because only the Stokes $I_{\lambda}$ and $V_{\lambda}$ profiles of spectral lines could be measured. The polarimetric sensitivity suffered from the determination of the $V$ profiles from $(I+V)$ and $(I-V)$ images exposed on different parts of the CCD chip. There exist strong needs for observations of the spectral profiles of the full Stokes vector with higher sensitivity and without spurious signals from the measurements with two different parts of the CCD detector. Thus, a full Stokes polarimeter also was implemented in April 2007. It operates with modulation of the incoming polarised light by means of liquid crystals and with minimisation of seeing induced polarimetric crosstalk, using a (modified) Savart plate and two halves of the subsequent detector exposed simultaneously.

The modifications of the "Göttingen" FPI spectrometer are presented in the following Sect. 2. In Sect. 3, we outline first full Stokes observations with high spatial resolution, obtained with speckle methods. The analysis of the observations demonstrated a good spectropolarimetric performance of the new setup and gave valuable data on small-scale magnetic fields in the solar 
atmosphere. Thus, in Sect. 4, we discuss results from a pore, from faculae near the solar south pole, and from quiet disc centre of the Sun. Section 5 concludes this paper.

\section{Improvements of the two-dimensional "Göttingen" spectro-polarimeter}

\subsection{Narrow-band spectrometer}

Until the end of 2006, the spectrometer worked with one narrowband etalon with a spacing of the plates of $d=1.101 \mathrm{~mm}$ and with a broadband etalon with $d=0.125 \mathrm{~mm}$, the latter serving as an order sorter for the 1.101 FPI. The broadband etalon was replaced by a further narrow-band FPI with $d=1.408 \mathrm{~mm}$. The 1.1 and the $1.4 \mathrm{~mm}$ FPIs have free apertures of $\oslash=70 \mathrm{~mm}$, for future use with GREGOR (Volkmer et al. 2007), and possess high finesses, $\mathcal{F} \geq 40$. The etalons and their electronic controllers were fabricated by IC Optical Systems Ltd, UK.

The FPIs are running in tandem and are mounted in the collimated beam, close to an image of the telescope pupil. This has certain advantages and disadvantages compared with the telecentric mounting close to a focus of the telescope (Kneer \& Hirzberger 2001). In the telecentric mounting, tiny inhomogeneities on the etalon surfaces cause an orange peel pattern in the images, and etalons with large apertures are required for some sufficiently large FOV. For collimated beam mounting, the etalons need extremely high optical quality, and the wavelength shift of the transmission towards blue, compared to the beam incident perpendicularly onto the etalons, increases quadratically with the distance from the centre of the FOV.

Yet, when running two narrow-band etalons in tandem, or even three etalons as in the TESOS spectrometer (Tritschler et al. 2002), both mountings require efforts to suppress the inter-etalon reflexes (Kneer \& Hirzberger 2001). In the former setup, with a broadband FPI, this was slightly tilted to direct the reflexes from the narrow-band FPI outside of the FOV of the detector. This is not possible with two narrow-band FPIs. Instead, an absorbing filter is placed between the two etalons. Interference filters of $F W H M=6 \ldots 10 \AA$, which are needed in any case to select the proper wavelength range, have a transmission of $60 . .70 \%$, at most. Yet near a pupil image, they have to be of very high optical quality. And with a free aperture of $\oslash=70 \mathrm{~mm}$, they are expensive. Instead of the interference filter, we placed a neutral density $(N D)$ filter with transmission of $70 \%$ and with high optical quality (from Reichmann Feinoptik, Hamburg/Germany) between the etalons, at the expense of optimum transmission of the spectrometer. The absorption by the $N D$ filter is sufficient to reduce the inter-etalon reflexes to a large extent (Kneer \& Hirzberger 2001). We will demonstrate below, when discussing the spatial resolution of the observations, that the optical quality of the spectrometer has remained high.

Figure 1 shows in the left panel the theoretical, normalised transmissions of the $1.1 \mathrm{~mm}$ and $1.4 \mathrm{~mm}$ etalon alone (dashed and dot-dot-dot-dashed, respectively), and as well, in solid, the transmission of the total spectrometer including the inter-etalon reflexes after reduction with a 70\% ND filter. The wavelength is $6173 \AA$ near the Fe I line with a Landé factor $g=2.5$ used below for the first observations with this new setup. The adopted finesses were $\mathcal{F}=40$. At this wavelength, the full spectrometer has thus a $F W H M$ of $25.4 \mathrm{~m} \AA$ and a spectral resolution of 243000 .

Yet not only the FWHM is much smaller than for one narrowband etalon alone, but also the wings of the transmission curve are substantially lowered. This suppresses much better than in
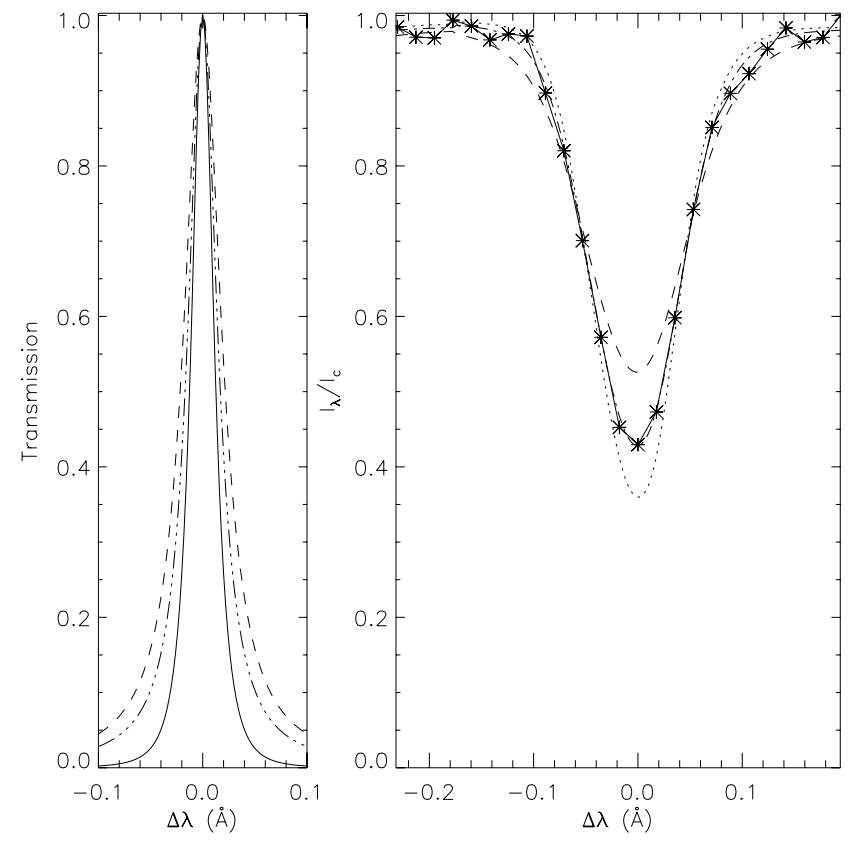

Fig. 1. Left panel: wavelength transmission of FPI spectrometer with finesses $\mathcal{F}=40$. Dashed: transmission of one etalon (spacing $1.101 \mathrm{~mm}$ ) alone; dot-dot-dot-dashed: transmission of $1.408 \mathrm{~mm}$ etalon; solid: transmission of total FPI setup. Right panel: average Fe I $6173 \AA$ line from quiet Sun disc centre. Solid: observed in this work, with asterisks at the measured wavelength positions, with interval $\delta \lambda=17.74 \mathrm{~m} \AA$; dotted: from Fourier Transform Spectrometer (FTS) atlas (Brault \& Neckel, quoted by Neckel 1999); dash-dotted: FTS profile convolved with the transmission curve of the spectrometer with two FPI etalons; dashed: FTS profile convolved with the spectrometer transmission before the upgrade.

the former setup the light from these wings. That is, when observing near the centre of a line, the (false) light from the continuum is strongly suppressed.

The right panel of Fig. 1 gives average disc centre profiles of the Fe I $6173 \AA$ line. The dotted profile is taken from the Fourier Transform Spectrometer (FTS) atlas (Brault \& Neckel, quoted by Neckel 1999). We adopt this as the "true" solar profile. The dashed profile is the FTS profile convolved with the Airy transmission curve of the spectrometer with only the $1.1 \mathrm{~mm}$ etalon (cf. the dashed curve in the left panel of Fig. 1). The dash-dotted profile represents the FTS profile convolved with the transmission curve of the combined 1.1 and $1.4 \mathrm{~mm}$ etalons (solid curve in left panel). The asterisks “*”, connected by solid straight lines, are the observed intensities, averaged over a FOV of $31^{\prime \prime} \times 52^{\prime \prime}$ and normalised to the same continuum level as the FTS profile. The scanning interval is $\delta \lambda=17.7 \mathrm{~m} \AA$.

The observed profile behaves close to expectation. It exhibits, however, fluctuations with a period of $\sim 60 \mathrm{~m} \AA$, best seen in the continuum. They are also present in data from the TESOS FPI spectrometer and termed "ghosts" by Tritschler et al. (2002). After many tests, we are certain that these do not stem from interferences at optical surfaces, nor from mistuning of the two etalons against each other. The producing company is yet searching for their reason and thinking of a remedy. All positions within the FOV are affected, i.e. the whole detector surface sees the modulation simultaneously with the same relative amount. Tests have shown that this pattern is stable in wavelength amplitude and with time, i.e. does not vary above the detection limit within a series of hundred scans. Thus, the effect on the 
polarimetric measurements is small. Efforts are underway to remove the modulations, e.g. via filtering. At present, we use the data as presented in Fig. 1.

\subsection{Full stokes polarimeter}

\subsubsection{Ferroelectric liquid crystals - FLCs}

Fortunately, we received two ferroelectric liquid crystals (FLCs) on loan from the Instituto de Astrofísica de Canarias. They serve as retarders for the subsequent linear polarisers (Savart plate, cf. Sect. 2.2.2 below). The orientation of their fast axes can be changed rapidly, in the $\mathrm{kHz}$ range, which is important for observation with speckle methods. The disadvantages of FLCs compared with nematic liquid crystals are their fixed retardance, independent of the applied voltage $(>5 \mathrm{~V})$, and their usefulness for only a limited wavelength range per FLC pair.

Each FLC is characterised by two states, i.e. two possible orientations of the fast axis. Combining the two states of the two FLCs, a cycle of modulation of the incoming Stokes vector gives four images (four exposures) on each of the two channels after the Savart plate. The nominal retardance of the first FLC is $\lambda / 2$, and that of the second FLC is $\lambda / 4$. The change of the axis orientations is, desirably, $45^{\circ}$. However the actual values, measured in the optical laboratory in our institute, differ from the nominal ones. Once in the optical laboratory of the Göttingen FPI in the VTT, we optimised experimentally the total polarimetric system by exposing it to well defined linearly and circularly polarised light at the wavelength selected for the solar observation.

The incoming Stokes vector $S$ is changed by the polarimeter's (calibrated) modulation matrix $\boldsymbol{M}$ leading to the measured four-vector $\boldsymbol{I}$. The Stokes vector is retrieved by inversion, i.e.

$\boldsymbol{I}=\boldsymbol{M} \cdot \boldsymbol{S} \quad \Rightarrow \quad \boldsymbol{S}=\boldsymbol{D} \cdot \boldsymbol{I}=\boldsymbol{M}^{-1} \cdot \boldsymbol{I}$.

The efficiencies of the device are defined as (e.g. Collados 2007)

$\varepsilon_{j}=\left(4 \sum_{i=1}^{4} \boldsymbol{D}_{j i}^{2}\right)^{-1 / 2}$,

where $j$ denotes any of the four Stokes parameters $(I, Q, U, V)$, and

$\varepsilon_{\mathrm{pol}}=\sqrt{\varepsilon_{Q}^{2}+\varepsilon_{U}^{2}+\varepsilon_{V}^{2}}$.

The empirical modulation scheme used in this work has, at $6170 \AA$, the efficiencies in the range

$\varepsilon_{I}=0.89, \varepsilon_{Q}=0.55$,

$\varepsilon_{U}=0.19, \varepsilon_{V}=0.54$

$\varepsilon_{\text {pol }}=0.75$.

The modulator system and the following Savart plate give, to each half of the detector, oppositely oriented polarisation with modulated amplitude. In the ideal case, upon modulation, the two halves change their role with regard to the signs of $Q, U, V$ in the measured combination of the Stokes components (in short: beam exchange). The modulation scheme has then a low $I \rightarrow$ $Q, U, V$ crosstalk. We demonstrate this for simplicity for the idealised case that one measures $(I \pm V) / 2$ at subsequent instants with one half of the detector and $(I \mp V) / 2$ on the other half, similarly as Collados (1999). After the light has passed the linear polariser (Savart plate), one measures the intensities $I^{+}\left(t_{1}\right), I^{+}\left(t_{2}\right)$, $I^{-}\left(t_{1}\right)$, and $I^{-}\left(t_{2}\right)$ in the "channels" "+" and "-" at the times $t_{1}$ and $t_{2}$ of the two cycle steps, respectively. The " + " channel gives

$I^{+}\left(t_{1}\right)=(I+V) / 2$,

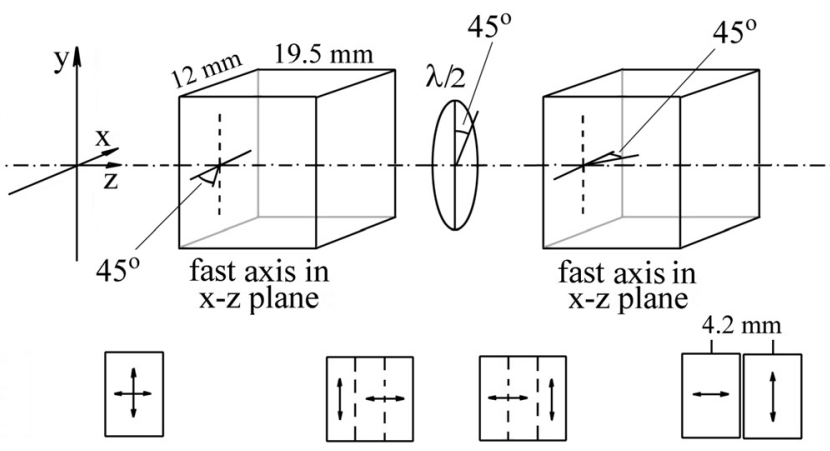

Fig. 2. Scheme of modified Savart plate, after Keller (2006): two calcites with a $\lambda / 2$ retarder in between. The line on the bottom depicts the orientation and separation of the linearly polarised beams.

$I^{+}\left(t_{2}\right)=(I+\delta I-V-\delta V) / 2$

where $\delta I$ and $\delta V$ account for seeing-induced crosstalk since the two images are not obtained strictly simultaneously. Similarly, the "-" channel gives

$I^{-}\left(t_{1}\right)=\left(1+\delta_{\mathrm{g}}\right)(I-V) / 2$,

$I^{-}\left(t_{2}\right)=\left(1+\delta_{\mathrm{g}}\right)(I+\delta I+V+\delta V) / 2$.

Here, $\delta_{\mathrm{g}}\left(\sim 10^{-2}\right)$ accounts for gain-table uncertainties and misalignments of the two channels. Combining Eqs. (4) to (7) one arrives at

$I_{\text {meas }}=\left(1+\delta_{\mathrm{g}} / 2\right)(I+\delta I / 2)+\delta_{\mathrm{g}} \delta V / 4$,

$V_{\text {meas }}=\left(1+\delta_{\mathrm{g}} / 2\right)(V+\delta V / 2)+\delta_{\mathrm{g}} \delta I / 4$.

The remaining contribution of the $I \rightarrow V$ crosstalk, thus determining the polarimetric sensitivity, is the last term of Eq. (9). It depends on the seeing conditions and can be estimated from measurements in the continuum near the observed spectral line. In most cases, $\delta_{\mathrm{g}} \cdot \delta I /(4 I) \leq 10^{-3}$. It is largest where the changes of $I$ are largest in the FOV, that is in the flanks of a line at large velocity gradients. Yet structures on the Sun with large velocity changes across the FOV are also those with most interesting dynamic and magnetic properties.

\subsubsection{Modified Savart plate}

The credit to use a modified Savart plate must go entirely to Keller (2006, private communication). We discuss it here shortly because the modification turned out to be important and because we consider it a good way to overcome the problem of astigmatism from the classical Savart plate.

Commonly, to have the costs low with elements of not highest optical quality, polarimetric devices are mounted in the convergent beam in front of the detector. Convergent rays passing obliquely a plane parallel plate suffer from astigmatism, which is the larger the smaller the focal ratio. Thus, images from the extraordinary beams in a Savart plate are affected by astigmatism. In a classical Savart plate, the astigmatic lines of the two beams are oriented perpendicularly to each other.

This is different for the modified Savart plate as shown in Fig. 2. The incoming light may be considered as the superposition of two linearly polarised rays. The first calcite separates the ordinary and extraordinary beams such that, seen into the direction towards the source, the vertically polarised beam is 
Table 1. Scanning parameters.

\begin{tabular}{cccccc}
\hline \hline & $r_{0}[\mathrm{~cm}]$ & $\delta \lambda[\mathrm{m} \AA]$ & \# Cycles & \#Sp. Pos. & $\Delta t[\mathrm{~s}]$ \\
\hline Pore & $\sim 16$ & 23.6 & 8 & 19 & $\sim 36$ \\
PFe & $12-14$ & 11.8 & 16 & 36 & $\sim 36$ \\
QS & $\sim 19$ & 17.7 & 6 & 25 & $\sim 36$ \\
\hline
\end{tabular}

displaced by approximately $2.1 \mathrm{~mm}$ (in the visible range). The following $\lambda / 2$ retarder plate rotates the orientations of the polarisation by $90^{\circ}$. For the second calcite, the formerly ordinary ray is now the extraordinary ray. Both beams suffer from the same amount of astigmatism with parallel oriented astigmatic lines. This can be removed with a cylindrical lens. Besides, the splitting is a factor $\sqrt{2}$ larger than for the classical case, the calcites are thus less expensive. The elimination of the astigmatism by a cylindrical lens will be important when this spectrometer will be mounted at the $1.5 \mathrm{~m}$ GREGOR telescope. In the present setup in the VTT, a cylindrical lens has not (yet) been implemented. According to ray tracing calculations (Soltau 2006, private communication), the astigmatism of the modified Savart plate gives a Strehl ratio still larger than 0.8 which allows still an excellent image sharpness. With a traditional Savart plate the astigmatism is larger by a factor of $\sqrt{2}$. In addition, the optics between the AO's wavefront sensor and the FPI spectrometer introduce an astigmatism of the same amount as that of the original Savart plate, but additive in one channel of linearly polarised light and subtractive in the other channel. There was thus, with a conventional Savart plate, no chance to place the detector at the position of the circle of least confusion simultaneously for both channels.

\subsubsection{Implementation in FPI spectrometer}

Each modulation cycle of the polarimeter consists of four images from the four combinations of orientation of the retardance axes. To minimise seeing induced crosstalk the four images are taken one immediately after the other. At frame-rates of 10-20 images/s, one cycle is completed within 200-400 ms. For spectro-polarimetric speckle imaging, 4-10 modulation cycles are recorded at each wavelength position during scanning.

\section{Observations and data analysis}

\subsection{Observations}

First observations after implementation of the new features of the spectropolarimeter were obtained during a testing period March 30 to April 4, 2007, at the VTT, Observatorio del Teide, Tenerife. They were supported by the Kiepenheuer Adaptive Optics System (KAOS, von der Lühe et al. 2003). Several short time sequences of spectropolarimetric scans were taken from various solar regions: activity with a pore at $\mu=\cos \vartheta=0.76$, polar faculae $(\mathrm{PFe})$ near the south pole at $(L, \psi)=\left(-11^{\circ},-72^{\circ}\right)$ corresponding to $\mu=0.41$, and quiet Sun disc centre. The seeing quality, albeit good, varied from day to day. The exposure times were always $10 \mathrm{~ms}$, giving a frame rate of $16.5 / \mathrm{s}$. The other scanning parameters, i.e. Fried parameter $\left(r_{0}\right)$ estimated by KAOS, spectral scanning step $(\delta \lambda)$, number of polarimetric cycles, number of spectral positions along the scanning range and duration of one scan $(\Delta t)$, were varied (see Table 1$)$.

From the same solar regions, broadband images at $630 \mathrm{~nm}$ $(F W H M=5 \mathrm{~nm})$ were recorded strictly simultaneously with the spectropolarimetric images. In addition, dark frames and flat field scans with moving telescope pointing and with defocussed telescope were obtained.

\subsection{Data analysis}

Dark corrections and flat fielding were applied. The broadband frames were reconstructed with the "Göttingen" speckle code (de Boer 1996), which uses the spectral ratio method (von der Lühe 1984) and speckle masking (Weigelt 1977). The speckle reconstruction takes into account that the image correction by KAOS varies with distance from the AO lockpoint. Thus, the variation of the Fried parameter across the field of view is fitted by a Gaussian centred in the lockpoint. Such distribution is then used to apply the proper speckle transfer function to each isoplanatic patch (cf. Bello González et al. 2007).

Differently from the notations above for the Stokes components, we denote here by lower case letters $2 \mathrm{D}$ arrays in the normal space and by corresponding upper case letters their Fourier transforms. From the $N=4 \ldots 8$ polarimetric cycles at each wavelength position and for each of the four components of the modulated Stokes vector, i.e. images $i_{i}(\lambda)$, exist $N$ speckle images $(i=1, \ldots N)$. The extension of the narrow-band speckle reconstruction (see e.g., Keller \& von der Lühe 1992; Krieg et al. 1999; Bello González et al. 2005) yields

$O_{\mathrm{n}, j}=H \frac{\sum_{i=1}^{N} I_{\mathrm{n}, j, i} I_{\mathrm{b}, i}^{*}}{\sum_{i=1}^{N}\left|I_{\mathrm{b}, i}\right|^{2}} O_{\mathrm{b}}$.

Here, $I_{\mathrm{b}, i}$ are (in the Fourier domain) the broadband speckle images taken simultaneously with the corresponding narrow-band images $I_{\mathrm{n}, j, i}, O_{\mathrm{n}, j}$ and $O_{\mathrm{b}}$ represent the reconstructed narrowband images of the component $j, j=1, \ldots 4$ and the reconstructed broadband image, respectively. The asterisk "*” denotes the conjugate complex and $H$ is a noise filter. Fourier transformation back to normal space gives the modulated Stokes images, which were then demodulated.

\subsection{Instrumental polarisation}

The telescopic crosstalk depends on the observing time, i.e. on the time during the day and on the date of observation. It has been taken into account by means of the polarisation model of the VTT elaborated by Beck et al. (2005b).

In principle, the polarisation properties of the optical components following the telescope (AO, $45^{\circ}$ mirrors, spectrometer) can be measured with a calibration unit provided for observation with the POLIS (polarimetric Littrow spectrograph) post-focus instrument (Beck et al. 2005a). This was not used, however, for these first observations due to a tight time schedule for testing.

Instead, for the latter optical parts, a correction of the $V \rightarrow$ $Q, U$ and $Q, U \rightarrow V$ crosstalks was performed with the following reasoning: we expect the strong $Q$ and $U$ profiles from the pore (amplitudes of $|Q|_{\max } \approx|U|_{\max } \leq 0.1 I_{\mathrm{c}}$, with $I_{\mathrm{c}}$ the continuum intensity), to be symmetric about line centre, and the $V$ profiles from the centre of the pore to be antisymmetric. Such properties were obtained by adding and subtracting certain amounts to and from the measured profiles, i.e.

$Q_{\text {corr }}(\lambda) \approx Q_{\text {meas }}(\lambda)-0.3 \cdot V_{\text {meas }}(\lambda)$

$U_{\text {corr }}(\lambda) \approx U_{\text {meas }}(\lambda)-0.3 \cdot V_{\text {meas }}(\lambda)$,

$V_{\text {corr }}(\lambda) \approx V_{\text {meas }}(\lambda)+0.3 \cdot Q_{\text {meas }}(\lambda)$, 
These corrections from the optics following the telescope are independent of the observing time and were applied to all $Q$, $U$, and $V$ measurements from the pore/activity region and the $\mathrm{PFe}$ region. It turned out to be a factor of approximately 4 larger than the correction for the telescopic crosstalk for Stokes $Q$ and a factor 2 larger for Stokes $U$. They were not applied to the disc centre observations. There, the $Q$ and $U$ profiles were small and will not be discussed here.

Figure 3 shows examples for $I, Q, U$, and $V$ profiles in the observed pore, from a $\mathrm{PF}$, and $V$ profiles from quiet Sun network and inter-network region at disc centre:

- upper panel: profiles from the inner pore (solid line) and surrounding magnetic features (dashed) show high-amplitude polarisation signals. The improved spectral purity allows to see the contribution of magneto-optical effects on the $V$ profiles from the pore;

- middle panel: polar faculae profiles show linear polarisation of the same order as the circular polarisation (solid). Some features from the surroundings with opposite polarity can be seen in circularly polarised light although they do not exhibit clear linear polarisation signal (dashed);

- lower panel: Stokes $V$ profiles of opposite polarity are easily found in the quiet Sun network at disc centre (left), and, looking in detail, signal with small amplitude from the internetwork with opposite polarities can also be seen.

\subsection{Scattered light}

Light scattered in Earth's atmosphere and by the telescope optics falsifies the signals, especially the low intensities measured in pores and umbrae of sunspots. The contribution from spurious light is approximated by (see, e.g., Martínez Pillet et al. 1997)

$I_{\mathrm{obs}}(\lambda)=(1-\alpha) I_{\text {true }}(\lambda)+\alpha \bar{I}_{\odot}(\lambda)$

or

$I_{\text {true }}(\lambda)=\left[I_{\text {obs }}(\lambda)-\alpha \bar{I}_{\odot}(\lambda)\right] /(1-\alpha)$.

Here, $\bar{I}_{\odot}$ represents the contribution from the ambient nonspot/pore region due to scattering and $\alpha I_{\text {true }}$ is the loss of intensity. For more discussions of the influence of scattered light see Kneer \& Mattig (1968), Martínez Pillet et al. (1997), and in work cited therein. The scattering parameter $\alpha$ was adjusted with the following reasoning: the $V$ profiles from the centre of the pore exhibit well separated extrema (and pronounced magnetooptic reversals between them), yielding maximum magnetic field strengths of $|B| \approx 2200 \mathrm{G}$. This measurement by separation of extrema is overestimated by few $100 \mathrm{G}$. The COG method gave a maximum value of $B_{\mathrm{LOS}} \approx 800 \mathrm{G}$. Equation (15) above was then applied to $I^{ \pm}(\lambda)=(1 / 2)[I(\lambda) \pm V(\lambda)]$ with the appropriate $\alpha$ that yielded $B_{\mathrm{LOS}} \approx \mu \times 2000 \mathrm{G}$ with $\mu=0.76$ from the heliocentric position of the pore.

The scattered light parameter was found in the range $\alpha=$ $0.30 \ldots 0.32$ which decreased the minimum continuum intensity in the pore $I_{\text {cont,min }}$ from approximately $0.5 \times \bar{I}_{\odot}$ to approximately $0.30 \times \bar{I}_{\odot}$, which is in good agreement with the value for large pores of $0.31 \times \bar{I}_{\odot}$ given by Sütterlin (1998). The scattered light correction was only applied to the pore region and there only to the Stokes $V$ profiles.
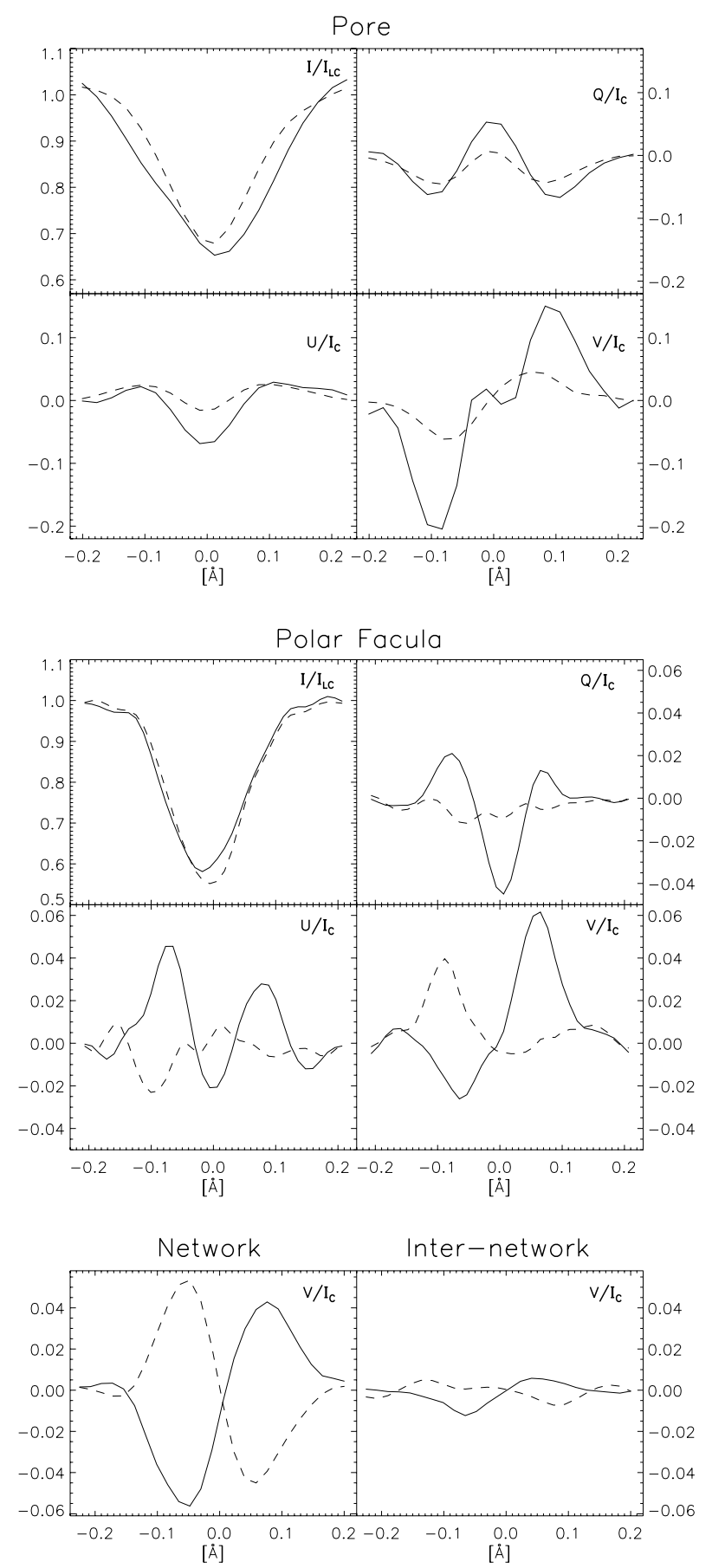

Fig. 3. Examples for $I, Q, U$, and $V$ profiles. From up to down: $I$ (normalised to local continuum for better comparison), $Q, U$, and $V$ profiles from the pore (solid) and surroundings (dashed), from the brightest polar facula (solid) and surrounding structure of opposite polarity (dashed), and from network (left) and inter-network (right) at quiet Sun disc centre.

\subsection{Physical parameters}

From the images $S(\lambda)$ we further derive the following parameters (for details see e.g., Bello González et al. 2005; Blanco Rodríguez et al. 2007):

1. The speckle-reconstructed broadband images and the reconstructed narrow-band images in the continuum serve as proxies for the temperature fluctuations in the continuum forming atmospheric layers. 

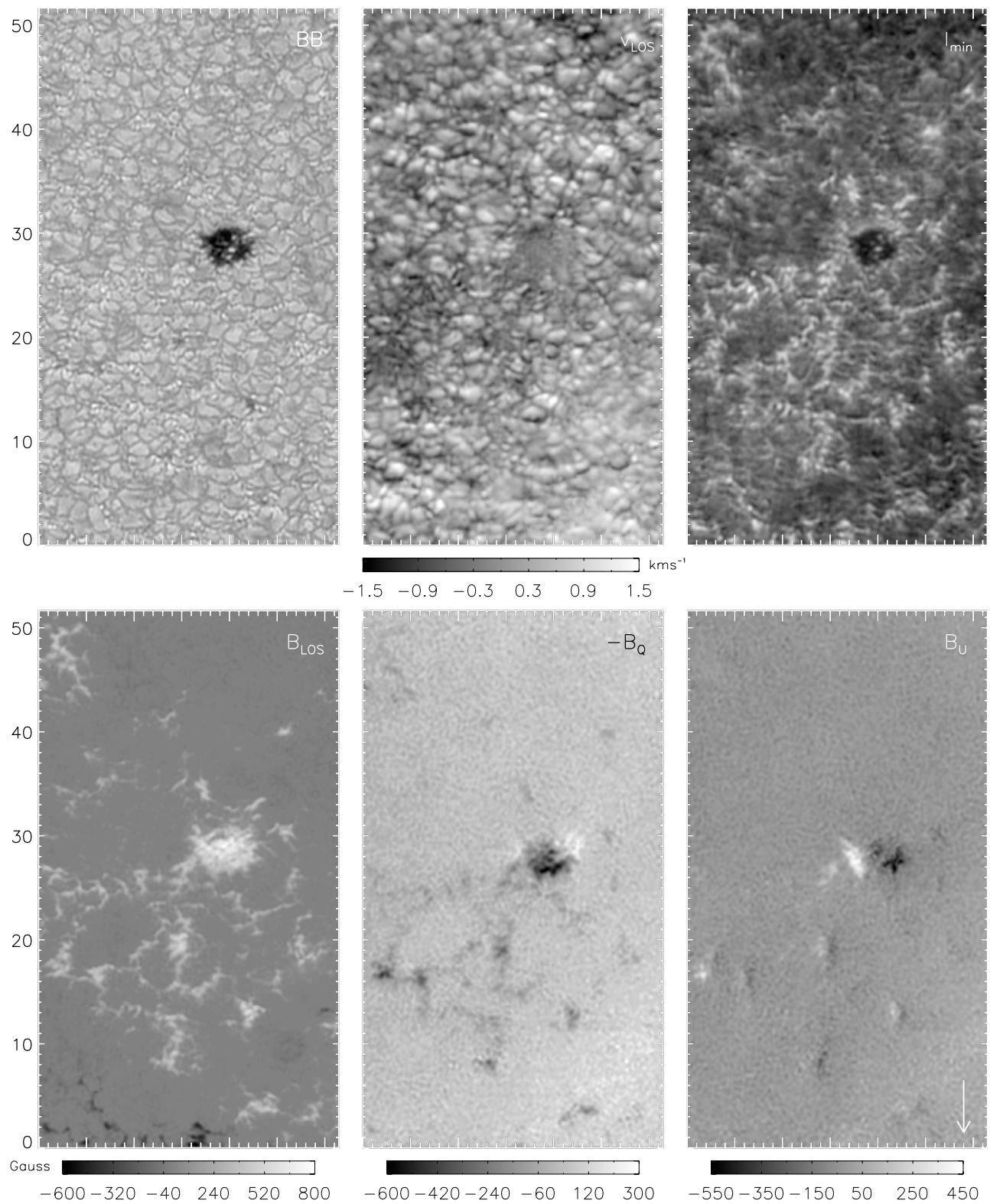

Fig. 4. Pore with activity at $\mu=0.76$. Upper row from left to right: broadband image, velocity determined from COG of Stokes I (reference wavelength: COG position of average $I$ profile), line minimum intensity; lower row: LOS component of magnetic field from the COG before correction for scattered light, component corresponding to Stokes $Q$ from WFA (positive $Q$ in the up-down direction, $-B_{\mathrm{Q}}$ is shown for presentation purpose), and component corresponding to $U$ (positive $U$ is along the $45^{\circ}$ direction clockwise from vertical). The arrow in Stokes $U$ (bottom right) points towards the limb. The presentation of the velocities is limited to $\pm 1.5 \mathrm{~km} \mathrm{~s}^{-1}$. The tickmarks are at a distance of $1^{\prime \prime}$.

2. The line minimum intensities were determined from parabolic fits to the minimum parts of the Stokes $I(\lambda)$ profiles.

3. Velocities were obtained from Doppler shifts of $I(\lambda)$ measured with the centre-of-gravity (COG) method. We take as reference wavelength for zero velocity the average COG position in the FOV.

4. The line-of-sight (LOS) component of the magnetic field was measured from the $V$ profiles with the COG method advised by Semel (1967) and Rees \& Semel (1979). This gives an average over the angular resolution element and over the formation height of the magnetic signal. The COG method measures the difference of wavelength position of the $\frac{1}{2}(I \pm V)$ profiles obtained from $I$ and $V$.

5. Finally, the magnetic field components perpendicular to the LOS were determined from fits to the $Q$ and $U$ profiles, also in the WFA (Landi Degl'Innocenti 1992, his Eqs. (11)). As this author comments, the formulae apply only in the WFA when using the centre values of the $Q$ and $U$ profiles, and an extension to the whole profiles has no physical justification. Tests using the analytical solution of the radiative transfer equation for a Milne-Eddington atmosphere have been performed. Values of the estimated magnetic field in the limit of the WFA, i.e. $\lambda_{B} \approx \Delta \lambda_{D}(\sim 800 \mathrm{G}$ for $\lambda 6173)$ are underestimated by $12 \%$. Yet, for strong magnetic fields, e.g. $\lambda_{B} / \Delta \lambda_{D}=2$, the magnetic field is underestimated by $23 \%$. Thus, we use the extension of the formulae as an estimate of the field components perpendicular to the LOS with reduced noise.

\section{Results}

\subsection{Pore and activity}

Figure 4 shows the speckle-reconstructed broadband image, the Dopplergram, the line minimum intensity, and the magnetic field components derived from $V, Q$, and $U$, from the whole FOV. The velocities will be discussed below in Sect. 4.4.

Obviously, when the field components perpendicular to the LOS are weak, the $Q$ and $U$ signals become noisy, especially in $U$ where the polarimetric efficiency was low. The magnetic fields in the $Q$ and $U$ images are signed to present their 

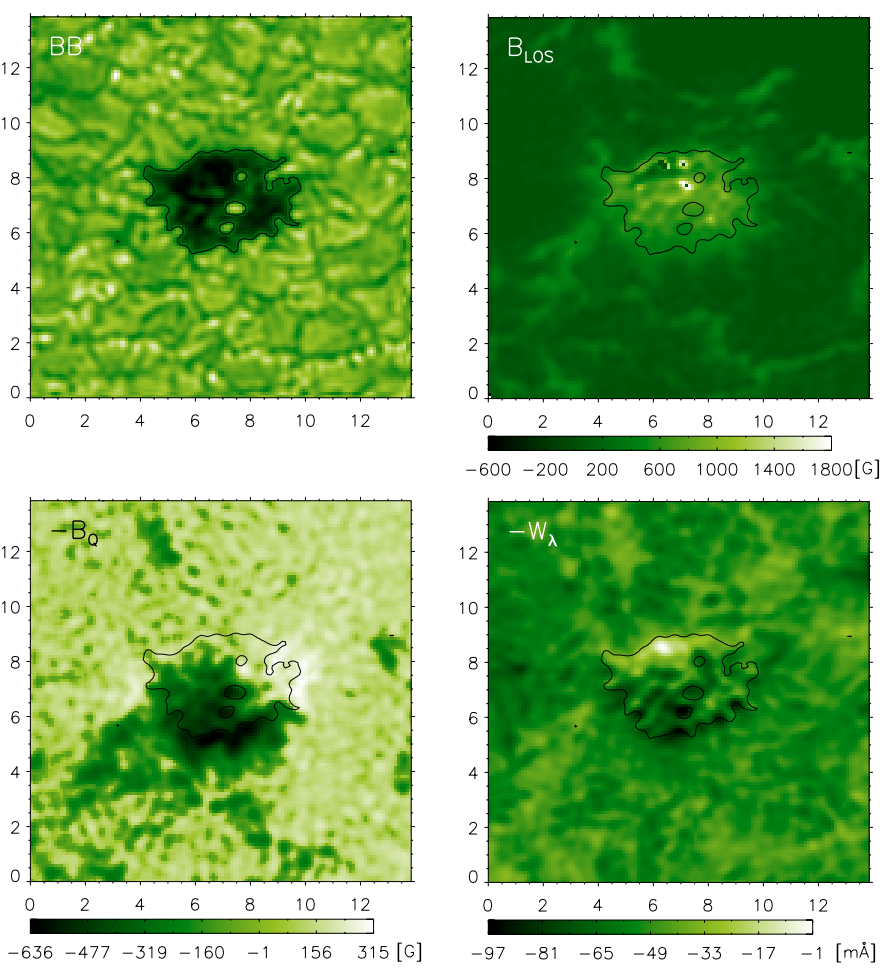

Fig. 5. Section of Fig. 4 showing the pore and its near surroundings. BB: broadband intensities; $B_{\mathrm{LOS}}$ : magnetic field strength determined with the COG method after correction for scattered light (set to $0 \mathrm{G}$ where COG failed due to the absence of absorption); $-B_{\mathrm{Q}}$ : field component perpendicular to LOS applying the WFA to $Q$ profiles (for presentation multiplied by -1$) ;-W_{\lambda}$ : negative values of equivalent width. The black contour outlines the $75 \%$ level of continuum intensity averaged over the ambient area. The axes are in arcsec.

orientation. It is possible to derive the magnetic field vector (within the known ambiguities) but not the scope of this study.

The broadband, line minimum, and $B_{\mathrm{LOS}}$ images exhibit strong inhomogeneities inside the pore. The facular points in the activity region surrounding the pore also are visible in detail in these images (see the online version for a high resolution presentation). On some locations, one starts to see, at $\mu=0.76$, the dark lanes in front (or above, in this image orientation) of the chains of facular points in both broadband and line centre images (see also Fig. 5). On the basis of $G$-band observations and their numerical simulations, these lanes were discussed by, e.g., Keller \& et al. (2004); Carlsson et al. (2004); Hirzberger \& Wiehr (2005).

The pore itself exhibits in its upper part a paradoxical $B_{\mathrm{LOS}}$ signal, as shown in more detail in Fig. 5. There, in a section of the FOV of Fig. 4, the broadband image and the LOS component and one component perpendicular to LOS of the magnetic field are presented. The black contour outlines the $0.75 \times \bar{I}_{\odot}$ intensity level. The signals from $Q$ and $U$ (latter not shown) behave as expected, with no to only weak signal on the upper ("northern") side of the pore where on expects the magnetic field pointing towards the observer.

Yet in this upper part and definitely inside the pore, the measured LOS magnetic field component is weak, even after scattered light correction. The scattered light is unpolarised, thus has no Stokes $V$ component. The lower right panel of Fig. 5 gives the reason for this behaviour. There, the equivalent widths $W_{\lambda}$ of the Fe I $6173 \AA$ line after scattered light correction are presented, for demonstration purpose the negative values of $W_{\lambda}$. In the upper part of the pore, the equivalent widths are very small, the line has almost disappeared. The $V$ profiles are as well very weak to absent. While in many parts of the pore the $V$ amplitudes amounted to $20 \%$ of the local continuum intensity, they were small in this special area before and after scattered light correction, smaller than $2 \%$. Apparently, the temperature gradient is very small in this region, thus no spectral line, including Stokes $V$ profile, is formed and there is no possibility to measure the magnetic field strength from $(I \pm V)$. This result and the small-scale structuring emphasise the highly dynamic properties of this pore.

\subsection{Polar faculae - PFe}

PFe were recently studied by Blanco Rodríguez et al. (2007). The reader may find in this work many citations to earlier work on this topic. In short, PFe are bright, small-scale structures and occur mainly during minimum of sunspot activity at the polar caps of the Sun at heliographic latitudes $|\psi| \geq 60^{\circ}$. They possess magnetic fields of kGauss strength.

Figure 6 shows an area from near the south pole with many $\mathrm{PFe}$ seen in the broadband and line minimum images. There are magnetic fields seen in the $Q$ and $V$ magnetic maps, i.e. magnetic field components perpendicular to the LOS, which are related to the brightest PFe. These field components are of the same order as the LOS components. Yet the maps are noisy and need more application of reduction methods. Here, we shall not discuss them further.

In the magnetogram, i.e. in the LOS field components from Stokes $V$, we find strong fields of positive magnetic polarity, as was the predominant global magnetic polarity near the solar south pole at the time when the observations were taken. They are related to the bright PFe. In addition, weaker fields of both polarities are found, partly as small-scale magnetic signals, partly with more diffuse appearance. As discussed by Blanco Rodríguez et al. (2007), not all magnetic structures are related to faculae and vice versa, not in all bright structures magnetic fields were detected. Presumably, the latter brightenings are granules with high intensity and little content of magnetic field.

In conclusion of this part on PFe, the application of full Stokes polarimetry with beam exchange turned out to be advantageous for the study of PFe. The magnetic sensitivity is much better than without beam exchange (cf. below Sect. 4.3), and the instrumental crosstalk could be taken into account, at least for the data presented here.

\subsection{Quiet Sun disc centre}

The magnetic fields in the quiet Sun, especially the inter-network (IN) magnetic fields, are of special interest since they harbour most of the (unsigned) solar magnetic flux and also are of importance for the dynamics of the solar atmosphere. Recent studies of IN fields, based on observations as well as on numerical simulations, may be found in Khomenko et al. (2003); Trujillo Bueno et al. (2004); Khomenko et al. (2005); Domínguez Cerdeña et al. (2006); Martínez González et al. (2007).

We present in Fig. 7 the 2D maps from a spectral scan in Fe I $6173 \AA$. The $Q$ and $U$ signals are very weak, as a result of the weakness of the horizontal field components. They are thus noisy and are not shown. To detect signals in $Q$ and $U$, at high spatial resolution, substantially more speckle images are needed. Observations performed with this aim in July 2007, but not yet analysed, may have the required low-noise quality. 

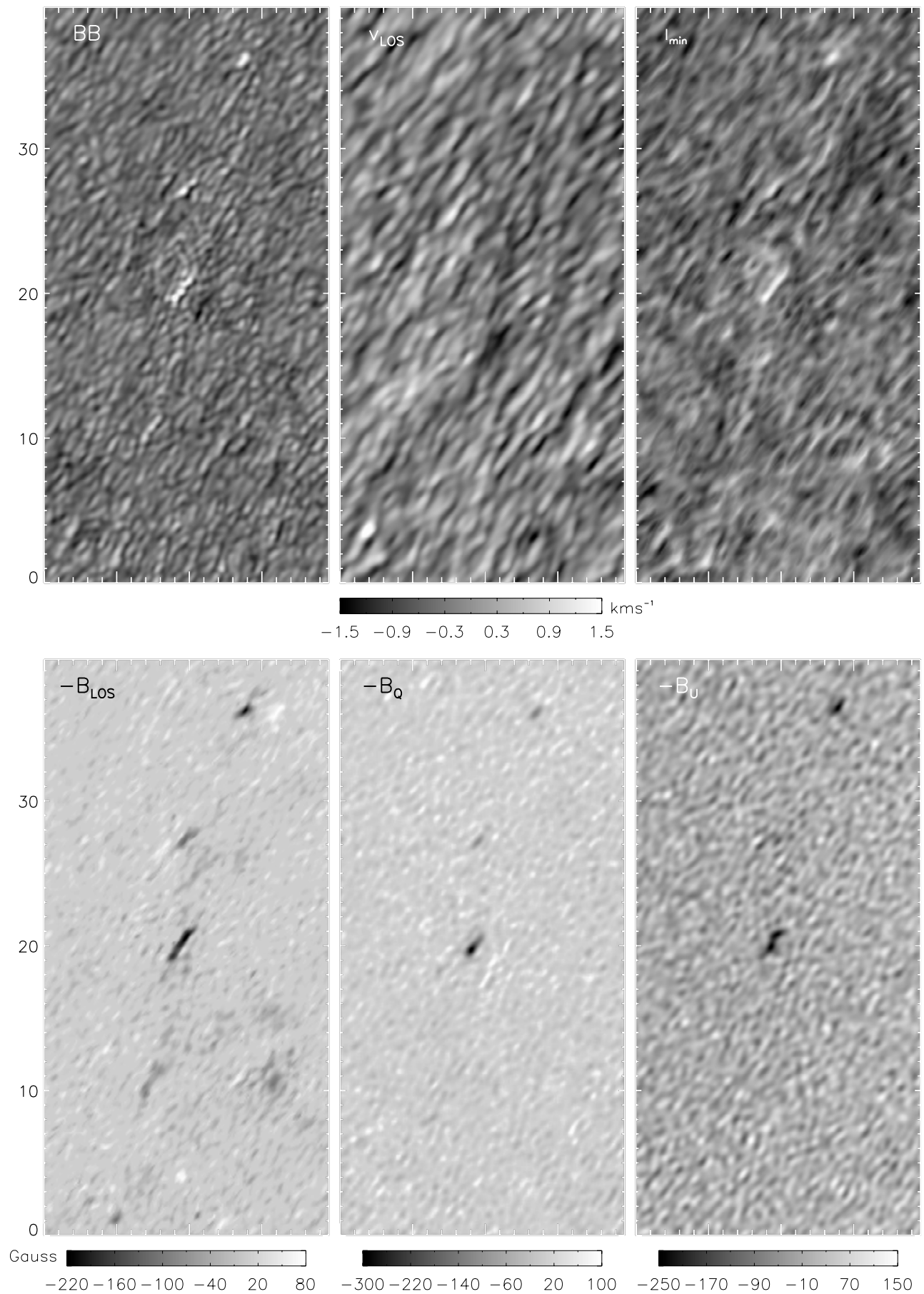

Fig. 6. Polar faculae near the south pole of the Sun at $\mu=0.41$. Arrangement of panels as in Fig. 4. A $20^{\prime \prime} \times 40^{\prime \prime}$ section of the full FOV is shown. Direction to limb is towards the upper left corner of the images. Positive $Q$ is top $\leftrightarrow$ bottom, positive $U$ under $45^{\circ}$ clockwise.
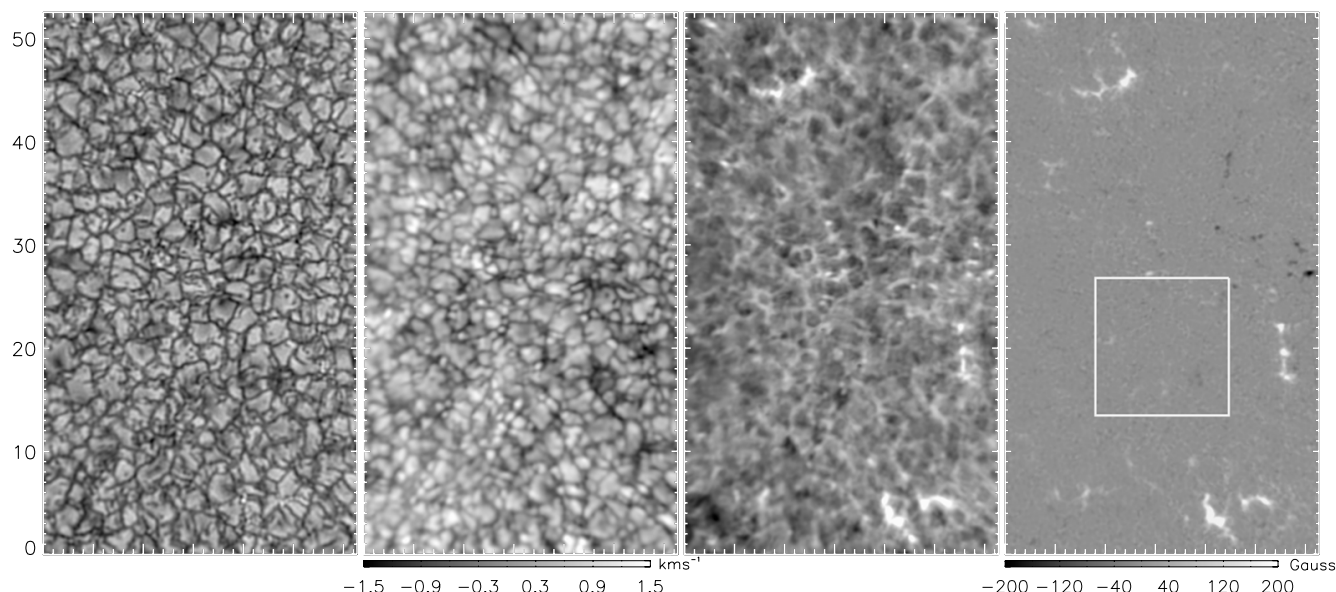

Fig. 7. Quiet Sun disc centre maps. From left to right: broadband image, Dopplergram, line minimum intensity, and LOS component of magnetic field. The $Q$ and $U$ signals are very weak and noisy, the corresponding maps of horizontal field components are thus not shown. Tickmark distances are 1". 

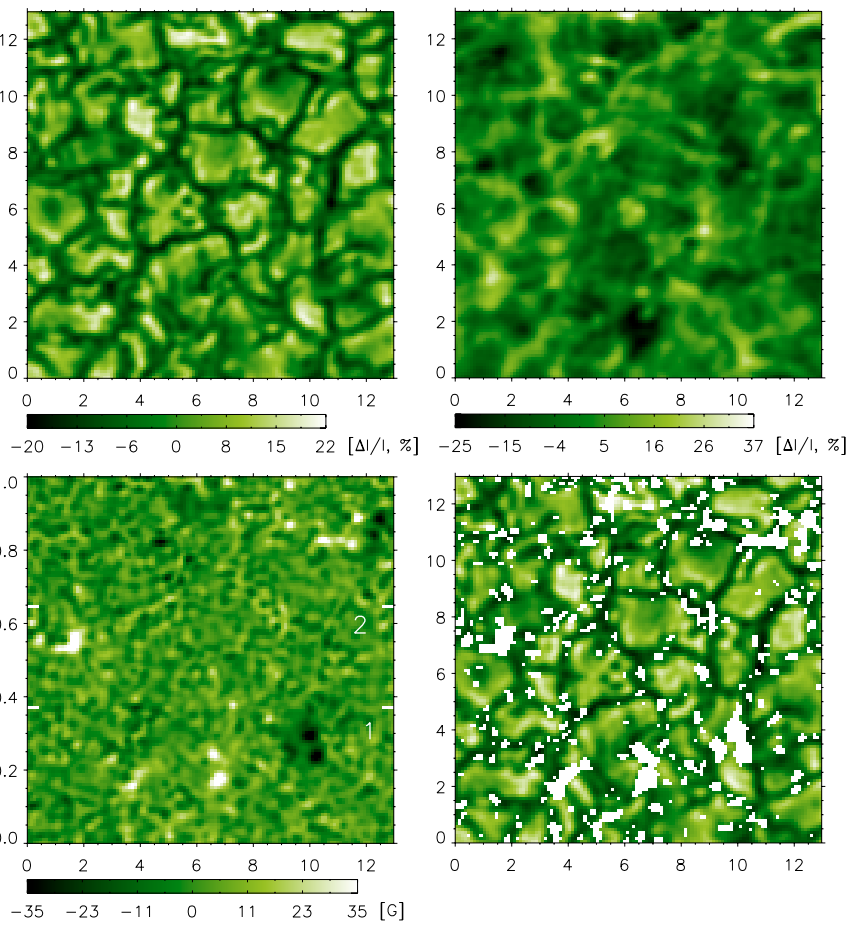

Fig. 8. Subfield of the observation at disc centre, marked by the white quadrat in the magnetogram of Fig. 7. Upper left: broadband image; upper right: line minimum image; lower left: magnetogram with $\left|B_{\mathrm{LOS}}\right|$ limited to $\leq 35 \mathrm{G}$; lower right: broadband image overlaid with a mask where $\left|B_{\mathrm{LOS}}\right| \geq 2 \sigma=11 \mathrm{G}$. The axes are in arcsec.

Figure 7 shows in the magnetogram (rightmost panel) network magnetic fields (upper left part, near right border, and lower right corner) which also are reflected in the line minimum intensities. There exist small-scale magnetic fields with LOS signals which are somewhat weaker than the clearly identified network fields. These too exhibit counterparts in the line minimum images. The network fields can also be traced to many tiny bright points in the speckle-reconstructed broadband images (see the online version for high resolution images). Due to the good spectral purity, the inverse granulation can clearly be seen in the line minimum image.

To study the IN magnetic fields in these observations, the section within the white rectangle in Fig. 7 is analysed. The section is shown in Fig. 8. The IN fields are emphasised by an appropriate limitation to low amplitudes.

While for the network the close correspondence of magnetic fields $\left|B_{\mathrm{LOS}}\right|$ and line minimum intensity $I_{\min }$ is obvious, a relation between IN $\left|B_{\mathrm{LOS}}\right|$ and $I_{\min }$ is not seen. Their correlation coefficient $C C$ is small, in the regions where $C C\left(I_{\min },\left|B_{\mathrm{LOS}}\right|,>\right.$ $11 \mathrm{G})=0.10$ counting only the positions where $\left|B_{\mathrm{LOS}}\right|>11 \mathrm{G}$.

Much of the pattern in the magnetogram of Fig. 8 stems from solar $V$ signals. The noise is difficult to determine, since many magnetic fields, although intermittent, are visible. From a magnetically "quiet" subarea in the centre of the FOV we obtain, as an upper estimate of the noise in $B_{\text {LOS }}$, the standard deviation $\sigma=5.5 \mathrm{G}$. The spatial resolution is good, 0.'30-0.'35 (see below). The polarimetric sensitivity at $3 \sigma$ is thus also good, $7-8 \times 10^{15} \mathrm{Mx}$.

The lower right panel of Fig. 8 presents the broadband granular image overlaid with a white mask for positions with $\left|B_{\mathrm{LOS}}\right|>$ $11 \mathrm{G} \widehat{\equiv} 2 \sigma$. The IN fields larger than this flux density are found predominantly in intergranular lanes, as found earlier, e.g. by Domínguez Cerdeña et al. (2003); Khomenko et al. (2003).

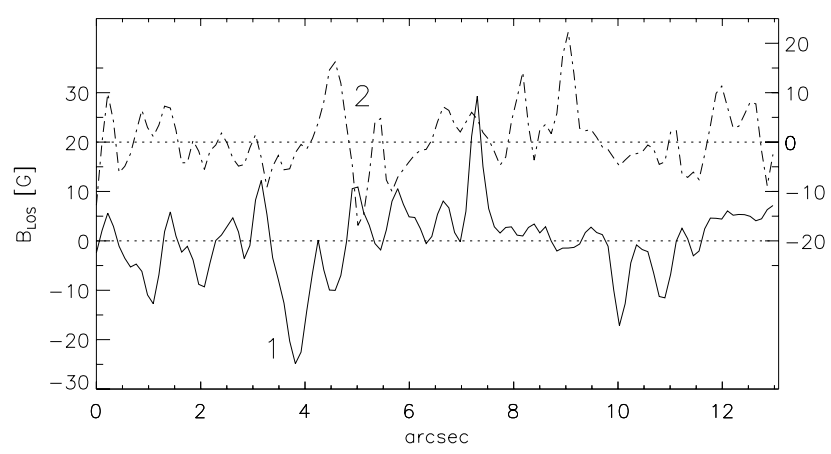

Fig. 9. Plots of LOS magnetic field components, determined with the COG method along the cuts in the magnetogram of Fig. 8 indicated by the short horizontal white lines. Solid and left ordinate: cut 1 in Fig. 8; dash-dotted and right ordinate: cut 2.

Taking only fields stronger than $2 \sigma$, we arrive at an average unsigned flux density $\left\langle\left|B_{\mathrm{LOS}}\right|\right\rangle=17 \mathrm{G}$ from $13 \%$ of the IN FOV. The signed flux density in these $13 \%$ of the selected IN FOV is $1.3 \mathrm{G}$, i.e. the negative and positive polarities are close to be balanced. Khomenko et al. (2003), from measurements in the Fe I infrared lines at $1.565 \mu$, find IN field strengths of $B \approx 250 \mathrm{G}$ with an area filling less than 0.02 in $50 \%$ of their observed IN area, i.e. a filling $<1 \%$ in the total FOV. For a comparison with the latter magnetic flux measurements, especially to obtain similar intrinsic field strengths, we have to assume that the average $B=17 \mathrm{G}$ measured in the present work comes from structures with a filling by magnetic fields of $7 \%$, due to still limited spatial resolution. Together with the $13 \%$ of area from above we arrive at an average field strength of approximately $240 \mathrm{G}$ in $0.9 \%$ of the observed IN FOV, values very similar to those given by Khomenko et al. (2003). This means that, irrespectively of the adopted filling factor, the total detected unsigned flux is the same as the one found by the latter authors.

Figure 9 demonstrates the high spatial resolution. It gives cuts along the lines indicated by short bars in the magnetogram of Fig. 8. Many of the small-scale magnetic structures exhibit a FWHM of approximately 0 !'33.

\subsection{Velocities}

In the above Figs. 4, 6, and 7, the reference wavelength for zero velocity is the COG position of the average intensity profile (from outside the pore for these observations). It thus contains the average granular blueshift with respect to the gravitational redshift (see e.g., Schröter 1957, especially his Fig. 9). With respect to this zero wavelength, the velocities inside the pore at $\mu=0.76$ show a redshift corresponding to $-0.43 \mathrm{~km} \mathrm{~s}^{-1}$, which is compatible with the picture that the gas inside the pore is at rest, on average.

Inspection of the velocities in the observed regions, pore/activity, $\mathrm{PFe}$, and quiet Sun disc centre, shows the influence of the 5-min oscillations overlaid on the granular convective flow. The presentation of the velocities is limited to $\pm 1.5 \mathrm{~km} \mathrm{~s}^{-1}$ in these Figs. 4, 6, and 7. The extrema are larger, however. They amount to $\pm 2.5 \mathrm{~km} \mathrm{~s}^{-1}$ with an rms value of $0.65 \mathrm{~km} \mathrm{~s}^{-1}$ for the disc centre and pore/activity data, and for the PFe region to $\left(-3.6 \mathrm{~km} \mathrm{~s}^{-1},+2.7 \mathrm{~km} \mathrm{~s}^{-1}\right)$ with an rms value of also $0.65 \mathrm{~km} \mathrm{~s}^{-1}$.

In Figs. 4 and 7, the granular flows exhibit a hilly structure, with smooth upflows and sharp lanes of downdrafts in between the latter, as found also in numerical simulations (e.g., Nordlund 


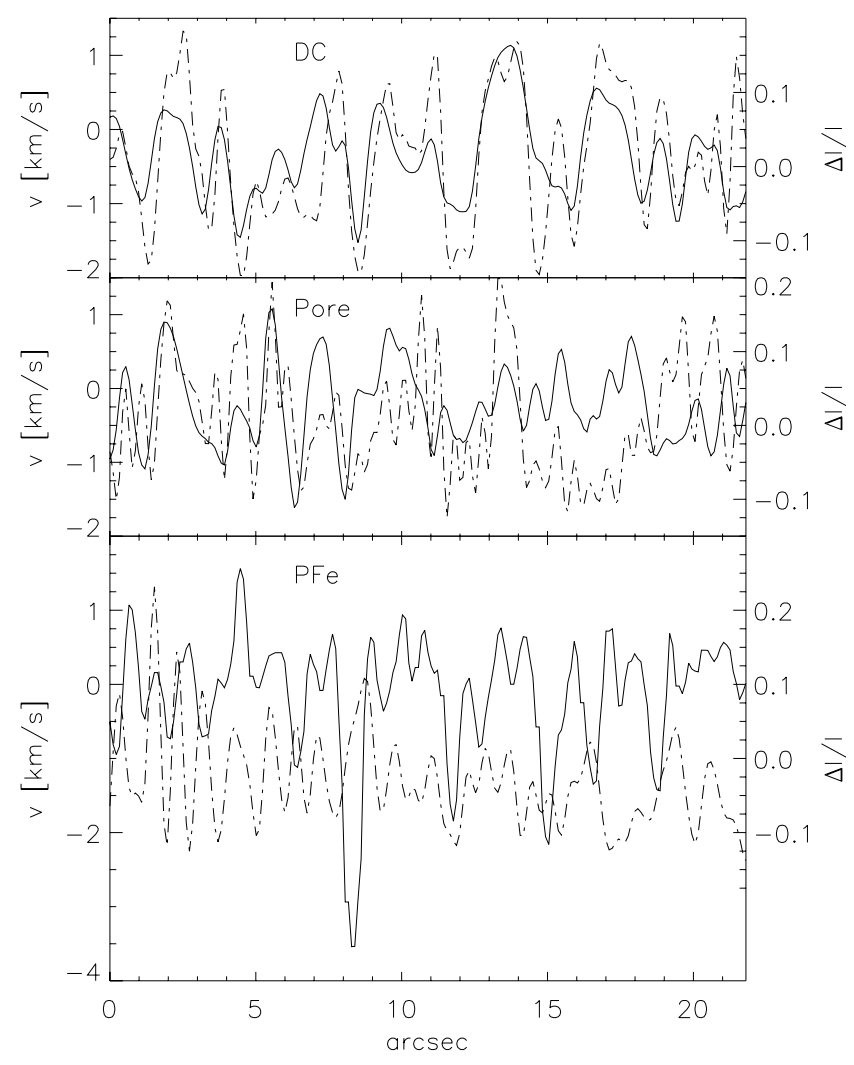

Fig. 10. Plots of LOS velocities and broadband intensity fluctuations from cuts of the respective maps at disc centre (upper panel, DC), near the pore from a part with only low magnetic activity at $\mu=0.76$ (Pore, middle panel), and from the area near the south pole at $\mu=0.41$ (PFe, lower panel). Solid: velocities, left ordinates; dash-dotted: broadband intensity fluctuations normalised to their average intensities, right ordinates.

et al. 1997). Figure 10 depicts velocity measurements together with the broadband intensities along cuts of $22^{\prime \prime}$ length in the three different areas. The disc centre observations exhibit a good correlation between granular velocity and broadband intensity. After subtraction of an image with boxcar smoothing over $3^{\prime \prime} 3^{2}$, to reduce the influence of the 5-min oscillations, the correlation coefficient is $C C=0.64$. With decreasing distance to the limb, the correlation breaks down. We obtain coefficients of 0.34 and 0.30 for the pore/activity observations and the PFe data, respectively. This is also reflected in the plots of Fig. 10.

The flows near the limb can attain at some positions very high velocities towards and away from the observer, up to $3 \mathrm{~km} \mathrm{~s}^{-1}$ and more. We conjecture that, near the limb at $\cos \vartheta=$ 0.41 , these velocities correspond to high-speed horizontal flows out of granules. On the basis of numerical simulations and analyses of strong profile asymmetries, it was discussed earlier by Cattaneo et al. (1990), Nesis et al. (1992) and Solanki et al. (1996) that these flows may occasionally become supersonic upon adiabatic gas expansion and thus cause a drop in temperature.

\section{Conclusions}

We have presented further improvements of the "Göttingen" FPI spectrometer, which allows short exposure times in the range of
10-20 ms and is thus an ideal instrument for speckle observations to yield high spatial resolution:

1. The implementation of a second narrow-band Fabry-Perot etalon, with a plate spacing of $1.408 \mathrm{~mm}$, resulted in a good spectral purity with approximately $25 \mathrm{~m} \AA F W H M$ in the visible spectral range and only small intensity contributions from the wings of the transmission curve.

2. By means of ferroelectric liquid crystals with short switching time, it has become a post-focus instrument with good capacity to measure small-scale magnetic fields on the Sun.

3. The use of a modified Savart plate allows to reduce substantially the astigmatism produced by classical Savart plates.

4. The instrument is fast, it takes scans of reasonable FOVs across a spectral line within 15-60 s, depending on requirements.

First observations with the new setup were presented bearing on the small-scale magneto-dynamics in the solar atmosphere:

1. The first target was a pore on the Sun exhibiting small-scale structures, with the surrounding activity. Stokes $I, Q, U$, and $V$ profiles were obtained. We found that in some positions in the pore there is almost no line depression. We interpret this result as a low atmospheric temperature gradient.

2. Faculae near the solar south pole $(\mathrm{PFe})$ were observed. The results of the analysis demonstrated the possibility for full Stokes polarimetry with high spatial resolution.

3. The results of polarimetric observations of an inter-network region show a spatial resolution 0. 30-0!'35 of the magnetogram and a good polarimetric sensitivity. The noise level amounts to approximately $5 \mathrm{G}$ at this resolution. The internetwork fields are mainly located in intergranular lanes. Despite the high spatial resolution, more total magnetic flux was not detected than in earlier observations with resolution of the order of $1^{\prime \prime}$.

More observations have been and will be taken. These include a measurement of the instrumental crosstalk, which could only be estimated post factum in a qualitative manner. Efforts are undertaken to see still weaker fluxes in the inter-network regions of the Sun and to obtain also observations of the horizontal components of the inter-network regions. We have the concept to improve steadily the spatial resolution and the polarimetric sensitivity. We expect to arrive at a stage where one starts resolving structures at the "large-scale" end of the magnetic field distribution, in which the fully entangled, turbulent fields form the "small-scale" end.

The "Göttingen" FPI spectrometer/polarimeter is in regular operation since many years. It is fast, and allows the study of small-scale structures on the Sun. In due time, it will be transferred, with a new name, to the $1.5 \mathrm{~m}$ solar telescope GREGOR, which is presently under construction at the Observatorio del Teide. There, the spectrometer with its high potential and versatility, will lead to best results on the dynamics of the solar magnetism.

Acknowledgements. We thank the solar physics group of the Instituto de Astrofísica de Canarias, especially M. Collados for giving us on loan the ferroelectric crystals and for the extensive help with the calibration. K. G. Puschmann, T. Seelemann, and W. Steinhof are thanked for the electronic polarimeter control and its implementation in the spectrometer software. C. Beck is thanked for providing IDL routines to model the telescopic polarisation. N.B.G. acknowledges financial support by Deutsche Forchungsgemeinschaft through grant KN 152/311. The Vacuum Tower Telescope is operated by the Kiepenheuer-Institut für Sonnenphysik, Freiburg, at the Spanish Observatorio del Teide of the Instituto de Astrofísica de Canarias. 


\section{References}

Beck, C., Schmidt, W., Kentischer, T., \& Elmore, D. 2005a, A\&A, 437, 1159

Beck, C., Schlichenmaier, R., Collados, M., Bellot Rubio, L., \& Kentischer, T. 2005b, A\&A, 443, 1047

Bello González, N., Okunev, O. V., Domínguez Cerdeña, I., Kneer, F., \& Puschmann, K. G. 2005, A\&A, 434, 317

Bello González, N., Kneer, F., \& Puschmann, K. G. 2007, in Modern Solar Facilities - Advanced Solar Science, ed. F. Kneer, K. G. Puschmann, \& A. D. Wittmann, Universitätsverlag Göttingen, 217

Bendlin, C., \& Volkmer, R. 1995, A\&AS, 112, 371

Bendlin, C., Volkmer, R., \& Kneer, F. 1992, A\&A, 257, 817

Blanco Rodríguez, J., Okunev, O. V., Puschmann, K. G., Kneer, F., \& S'anchez-Andrade Nuñ o, B. 2007, A\&A, in press

Borrero, J. M. 2007, A\&A, 471, 967

Borrero, J. M., Solanki, S. K., Lagg, A., Socas-Navarro, H., \& Lites, B. 2006, A\&A, 450, 383

Carlsson, M., Stein, R. F., Nordlund, Å., \& Scharmer, G. B. 2004, ApJ, 610, L137

Cattaneo, F., Hurlburt, N. E., \& Toomre, J. 1990, ApJ, 349, L63

Collados, M. 1999, in 3rd. Advances in Solar Physics Euroconference: Magnetic Fields and Oscillations, ed. B. Schmieder, A. Hofmann, \& J. Staude, ASP Conf. Ser., 184, 3

Collados, M. 2007, in Modern Solar Facilities - Advanced Solar Science, ed. F. Kneer, K. G. Puschmann, \& A. D. Wittmann, Universitätsverlag Göttingen, 143

Collados, M., Lagg, A., García Cíaz, J. J., et al. 2007, in The Physics of Chromospheric Plasmas, ed. P. Heinzel, I. Dorotovič, \& R. J. Rutten, ASP Conf. Ser., 368, 611

de Boer, C. R. 1996, A\&AS, 120, 195

Domínguez Cerdeña, I., Sánchez Almeida, J., \& Kneer, F. 2003, A\&A, 407, 741

Domínguez Cerdeña, I., Sánchez Almeida, J., \& Kneer, F. 2006, ApJ, 646, 1421

Gandorfer, A. 2003, Astron. Notes/AN, 324, 318

Hirzberger, J., \& Wiehr, E. 2005, A\&A, 438, 1059

Ichimoto, K., Tsuneta, S., Suematsu, Y., et al. 2004, SPIE, 5487, 1142

Keller, C. U. 2006, private communication

Keller, C. U., \& von der Lühe, O. 1992, A\&A, 261, 321

Keller, C. U., Schüssler, M., Vögler, A., \& Zakharov, V. 2004, ApJ, 607, L59

Khomenko, E. V., Collados, M., Solanki, S. K., Lagg, A., \& Trujillo Bueno, J. 2003, A\&A, 408, 1115

Khomenko, E. V., Martínez González, M. J., Collados, M., et al. 2005, A\&A, 436, L27

Kneer, F. 2007, in Modern Solar Facilities - Advanced Solar Science, ed. F. Kneer, K. G. Puschmann, \& A. D. Wittmann, Universitätsverlag Göttingen, 67
Kneer, F., \& Mattig, W. 1968, Sol. Phys., 5, 42

Kneer, F., \& Hirzberger, J. 2001, Astron. Notes, 322, 383

Koschinsky, M., Kneer, F., \& Hirzberger, J. 2001, A\&A, 365, 588

Krieg, J., Wunnenberg, M., Kneer, F., Koschinsky, M., \& Ritter, C. 1999, A\&A, 343,983

Landi Degl'Innocenti, E. 1992, in Solar Observations: Techniques and Interpretation, First Canary Islands Winter School, ed. F. Sánchez, M. Collados, \& M. Vázquez, Cambridge Univ. Press, Cambridge UK, 73

Martínez González, M. J., Collados, M., Ruiz Cobo, B., \& Solanki, S. K. 2007 A\&A, 469, L39

Martínez Pillet, V., Lites, B. W., \& Skumanich, A. 1997, ApJ, 474, 810

Martínez Pillet, V., Collados, M., Sánchez Almeida, J., et al. 1999, in High resolution solar physics: theory, observations and techniques, ed. T. R. Rimmele, K. S. Balasubramaniam, \& R. R. Radick, ASP Conf. Ser., 183, 264

Neckel, H. 1999, Sol. Phys., 184, 421

Nesis, A., Bogdan, T. J., Cattaneo, F., et al. 1992, ApJ, 399, L99

Nordlund, Å., Spruit, H. C., Ludwig, H.-G., \& Trampedach, R. 1997, A\&A, 328, 229

Puschmann, K. G., Kneer, F., Seelemann, T., \& Wittmann, A. D. 2006, A\&A, 445,337

Rees, D. E., \& Semel, M. D. 1979, A\&A, 74, 1

Scharmer, G. B., \& Spruit, H. 2006, A\&A, 460, 605

Schrijver, C. J., \& Title, A. M. 2003, ApJ, 597, L165

Schröter, E. H. 1957, ZAp, 41, 141

Semel, M. D. 1967, Ann. Astrophys., 30, 513

Solanki, S. K., Rüedi, I., Bianda, M., \& Steffen, M. 1996, A\&A, 308, 623

Solatu, D. 2006, private communication

Sütterlin, P. 1998, A\&A, 333, 305

Title, A. M., Frank, Z. A., Shine, R. A., et al. 1993, ApJ, 403, 780

Tritschler, A., Schmidt, W., Langhans, K., \& Kentischer, T. 2002, Sol. Phys., 211, 17

Trujillo Bueno, J., Shchukina, N., \& Asensio Ramos, A. 2004, Nature, 430, 326 Volkmer, R., Kneer, F., \& Bendlin, C. 1995, A\&A, 304, L1

Volkmer, R., von der Lühe, O., Kneer, F., et al. 2007, in Modern Solar Facilities Advanced Solar Science, ed. F. Kneer, K. G. Puschmann, \& A. D. Wittmann, Universitätsverlag Göttingen, 39

von der Lühe, O. 1984, J. Opt. Soc. Am. A1, 510

von der Lühe, O., Soltau, D., Berkefeld, T., \& Schelenz, T. 2003, SPIE, 4853, 187

Weigelt, G. P. 1977, Optics Comm., 21, 55

Westendorp Plaza, C., del Toro Iniesta, J. C., Ruiz Cobo, B., \& Martínez Pillet, V. 2001, ApJ, 547, 1148 